\title{
MRP OPTIMIZATION MODEL FOR A PRODUCTION SYSTEM WITH REMANUFACTURING
}

\author{
Fernanda M.P. Raupp ${ }^{1 *}$, Katharine De Angeli², \\ Guina G.S. Alzamora ${ }^{2}$ and Nelson Maculan ${ }^{3}$ \\ Received November 18, 2013 / Accepted May 2, 2015
}

\begin{abstract}
Science and technology practitioners have been studying how to take economical, social and environmental advantage of industrial residuals and discarded products. In this sense, this paper presents a Material Requirements Planning (MRP) optimization model for a particular production system that, besides manufacturing a final product and its main assembling component, it recovers units of the component from units of the returned product as well. It is assumed that the demand for the final product is independent of the amount of the returned product available, and that the market for the final product is not segmented. Considering that all parameters of the MRP mathematical model have deterministic nature, we prove that this production planning problem is NP-hard. We also show computational experiments with the model using an optimization solver and analyze some possible industrial scenarios, as well.
\end{abstract}

Keywords: MRP optimization model, remanufacturing, production planning.

\section{INTRODUCTION}

Nowadays industrial organizations are increasingly concerned on how to use or process industrial residuals and discarded products to avoid environmental pollution and waste of natural resources, to be more responsible with social issues, and, most of all, to take economical advantage by processing these items. Of course, these concerns are not isolated, but related. Therefore, managers have to make decisions considering broad and complex scenarios, specially, because they have to attend to exogenous pressures such as governmental regulations and customers desires with respect to environmental and social issues, and mainly endogenous pressure for being more economic efficient.

\footnotetext{
*Corresponding author.

${ }^{1}$ Laboratório Nacional de Computação Científica, Caixa Postal 95113, 25651-075 Rio de Janeiro, RJ, Brazil.

E-mail: fernanda@lncc.br

2 Pontifícia Universidade Católica do Rio de Janeiro, Departamento de Engenharia Industrial, Caixa Postal 38097, 22453-900 Rio de Janeiro, RJ, Brazil. E-mail: katharinedeangelih@gmail.com; guinas@ gmail.com

${ }^{3}$ Universidade Federal do Rio de Janeiro, COPPE-Engenharia de Sistemas e Computação, Caixa Postal 68511, 21941-972 Rio de Janeiro, RJ, Brazil. E-mail: nelson.maculan@gmail.com
} 
Returned products can have distinct origins, such as, for instance, manufacturing defect, obsolescence, material deterioration or lack of spare component, forcing companies to plan their operations with respect to the amount of product returns, see for example Reimann \& Zhang (2013).

The main concern is how to integrate product returns/residuals with the traditional forward supply chain. According to Thierry et al. (1995), depending on the quality and degree of disassembly of the returns/residuals, recovery operations are classified into repair, refurbishing, remanufacturing, cannibalization, and recycling.

Reverse Logistics (RL) is the field of science formally concerned with the study of moving goods from their typical final destination for the purpose of capturing value through a production process or proper disposal (Rogers \& Tibben-Lembke, 1999). Consolidated practice of reverse logistics can be found in the paper industry, for example. According to the American Paper Industry Association Council (2013), collected paper has been recycled at an increasing rate. One can observe that, in recent years, a lot of efforts have been made to adapt the paper production system to use discarded paper as raw material. Remanufacturing of tires is another good example of a common RL practice, which attends the demand of customers for retreated tires and of manufactures of tire-derived products, such as rubber floors and bricks.

Optimization models for manufacturing planning systems with recovering activities have appeared lately in the academic literature covering different aspects, including the Material Requirements Planning (MRP) model and its variations. In broad applications, MRP supports a large set of control functions in a multi-echelon production system, including order release (batching and timing), inventory management, material allocation and coordination, order tracking, and data management (Karmarkar \& Nambimadom, 1996).

Resembling the EOQ formula, existing studies present formulae with and without uncertain parameters. For instance, in Teunter (2004), the author considered two alternating policies to determine the lot-sizes of production and recovering with deterministic parameters. This classic problem was also extended with a remanufacturing option in the work of Helmrich et al. (2014), in which the authors propose mathematical models to remanufacture returned products or produce new items with separate or joint setup costs.

In the search for the best production planning, many studies considered multi-item, singlestage, and multi-period models, with and without uncertainty on the parameters. For example, in Bayindir et al. (2007), a single-period optimization model is presented for a production system with manufactured and remanufactured items, considering uncertain demands, capacity constraint and one-way substitution (the demand for the remanufactured item can be satisfied by the new manufactured item). In Inderfurth \& Jensen (1999), a quantitative model is presented considering a single-stage single-period system with recovery option, allowing remanufacturing of reusable products and components. Considering a scenario with uncertainty on demand, external return flows and lead time, Gotzel \& Inderfurth (2002) presented an extended Material Requirements Planning (MRRP) model for a hybrid single-stage multi-period production and 
remanufacturing system. Whereas in Schulz \& Ferretti (2011) disassembly operations, included in a specific recovery step, are treated in a stochastic environment. De Puy et al. (2007) proposed an approach to estimate the expected number of remanufactured units to be completed in each future period. The approach is a probabilistic form of standard MRP, it considers variable yield rates of good, bad, and repairable components that are harvested from incoming units, and probabilistic processing times.

According to Fleischmann (2000), the main issues of Production and Operations Management of RL are: (1) disassembly, (2) Material Requirements Planning (MRP) in a product recovery environment, and (3) scheduling remanufacturing operations. In respect to the second issue, the author stated that most of the works in the literature rely on a reverse bill of materials (BOM), due to the difficulties faced on the direct way. As we can see, in Barba-Gutiérrez et al. (2008), the disassembling production planning problem is addressed through a reversed form of the regular MRP (RMRP), considering independent sources of demand for the multiple components originated from disassembling a certain product over a time horizon. The problem objective is to determine the lot sizing of the disassembled components without producing excess inventories.

An innovative study is presented in Mitra (2012), where a deterministic and a stochastic model are proposed to evaluate optimal values for the inventory police parameters of a two-echelon closed loop supply chain, assuming that there is correlation between the product demand and the availability of the returned product. Finally, we call attention to the production planning models with remanufacturing and disposal activities proposed in Piñeyro \& Viera (2010, 2012) for particular production systems, which are single-stage uncapacitated lot-sizing models with deterministic parameters. Whereas the former work deals with two independent demand streams with one-way substitution, the latter generates production plans by fixing periods of the planning horizon for remanufacturing. Readers interested in a review of RL case studies may consult Brito et al. (2005), and Lage Junior \& Godinho Filho (2012).

In this work, we propose an optimization model for the Material Requirements Planning (MRP) of a particular multi-stage production system, that manufactures a finished item using a brand new or a recovered assembling component, among other provided inputs, with disposal option and capacity constraints. It can be considered as a step further from the models proposed in Piñeyro \& Viera (2010, 2012), as the planning of a capacitated multi-stage production system with remanufacturing and disposal activities is now addressed, considering a non-segmented market for the final product. The remanufacturing activities are processed in a intermediate phase, such that units of the recovered component could be used to assemble the final product. There is no external demand for the recovered component. Here, the BOM of the production system is handled as in traditional MRP. We show a novelty application of the traditional MRP in the context of procuring, manufacturing, recovering and disposal process.

Particularly, a multi-item, multi-stage and multi-period lot-sizing mathematical model for a particular production planning problem with remanufacturing and disposal options, and capacity constraints is proposed here. It is assumed that the demand for the final product is independent from the arrival of units of the returned product along the planning horizon, in the sense that 
there is no correlation between those actions, and that the market for the final product is not segmented, in the sense that customers could not differentiate a unit of the final product assembled with a brand new component from a unit assembled with a recovered one. Furthermore, to show that the production planning problem addressed here is difficult, we prove that the problem model is NP-hard, and present the response of the proposed planning model for distinct industrial scenarios of a particular academic instance, through computational experiments using CPLEX.

The paper is organized as follows. In Section 2, we describe the studied production planning problem, the planning decisions involved and the objectives to be achieved. In Section 3, we show the mathematical planning model, describing its parameters, decision variables, constraints and objective function and prove the problem model is NP-hard. Numerical experiments are showed in Section 4 together with some analysis generated from the consideration of distinct industrial scenarios. Final comments are presented in Section 5.

\section{PROBLEM DEFINITION}

Here, we address a particular multi-stage production planning problem with remanufacturing and disposal options, and capacity constraints. Remanufacturing is understood as the industrial process of recovering items from returned products, which ensures quality and functionality as brand new manufactured items. Figure 1 shows the bill of materials (BOM) related to the particular production system here addressed.

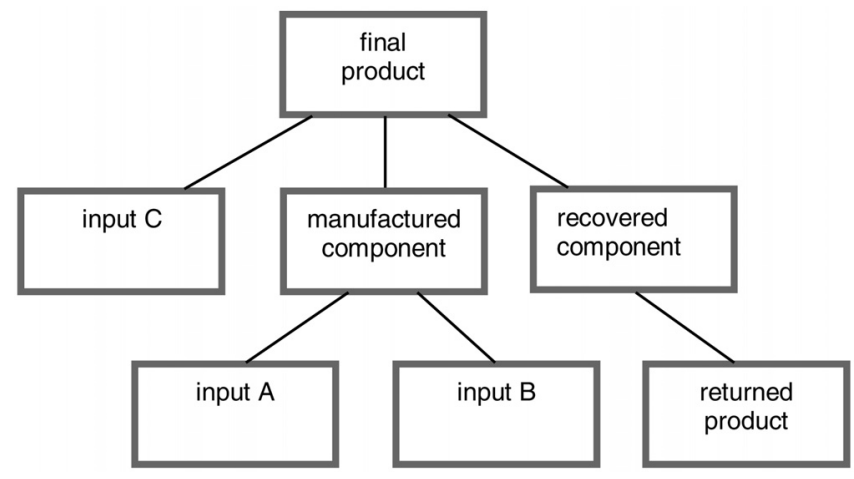

Figure 1 - Bill of materials of the production system with remanufacturing.

Actually, the particular production system has two distinct production lines. One for the manufacturing process, which shares the resources to produce new units of the main component as well as units of the finished item, and other exclusively for recovering units of the returned component. In the recovering process, units of the returned final product are disassembled in order to select and then repair the units of the main component therein. The remaining disassembled items are then discarded. Each unit of the returned product has one unit of the main component that is either remanufactured or discarded. Both production lines have capacity constraints, the first is given in terms of available time capacity and the second is given in terms of maximum units recovered. 
It is assumed that a known fixed average rate of units of the disassembled component, originated from units of the returned product, is not considered as input for the recovering process by not meeting the basic constitutive properties needed in the remanufacturing process, along the planning horizon. In Figure 2, we show the flow of acceptable assembling component units for the considered production planning. All the discarded items are accumulated in a tank, built specifically for this purpose, until its capacity is enough to load a garbage truck. No activities should be planned beyond the disposal.

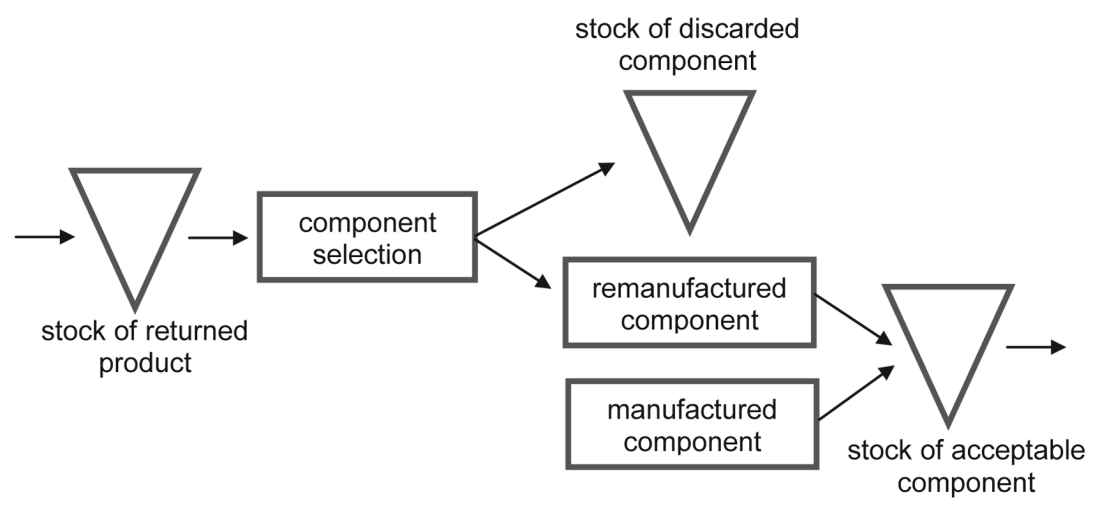

Figure 2 - Flow of acceptable assembling component units.

Also, storage capacity is considered for the stock of returned product, as well as for the stock of serviceable component and finished product. At the beginning of the planning horizon, there are no initial inventories of the items, except for the returned product, whose initial inventory level coincides with its maximal storage capacity.

A single source of external demand for the final product is considered, meaning that the market for the final product is not segmented, in the sense that customers could not differentiate a unit of the final product assembled with a brand new component from a unit assembled with a recovered one. (For instance, consider recovering metal hinges and handles from returned furniture, doors and gates to be used in new units of furniture, doors and gates.) The demand forecast in number of units for the final product per period is known for a coming short-term horizon. Also, independent from the demand for the final product, it is assumed that the amount of units of the returned product is known in advance for each period of the time horizon. There is no external demand for the recovered component.

\section{MRP OPTIMIZATION MODEL}

In this section, we present an MRP optimization model to support the decisions on the quantities and the timing of production of the final product and the main assembling component over a finite and discrete short-term horizon. Simultaneously, the model supports the decisions on the quantities and the timing of recovering the disassembled component, discarding the remaining 
of the disassembled components of the returned product, as well as procuring the inputs from suppliers needed in the production processes.

The Material Requirements Planning (MRP) model integrates the decisions on the production of finished items with the decisions on the procurement of all intermediate products and raw materials to satisfy costumer demand over a short or medium-term horizon. One of the advantages of the MRP optimization model over the MRP decomposition approach (the one that uses spreadsheets as resources) is the opportunity to have a global optimal production plan found by an exact solution method, whereas through the decomposition version a guaranteed feasible plan can be obtained by making use of expertise and/or heuristics.

The industrial activities that should be planned in the considered production system are: purchasing raw materials from external suppliers, manufacturing units of the finished item and new units of the component, remanufacturing units of the selected returned component, and discarding useless returned items. Procurement and production lead times, including the discarding lead time, are assumed constant along the planning horizon. They reflect the minimum time necessary for an activity to be concluded.

So, solving the MRP model will allow the determination of how much and when to procure/ produce/remanufacture/discard of each raw material/component/finished item over a short-time horizon.

Before presenting the MRP model, we show in Figure 3 the model structure based on BOM (already given in Figure 1), followed by the notation used hereafter for indices of sets, parameters and decision variables in the model.

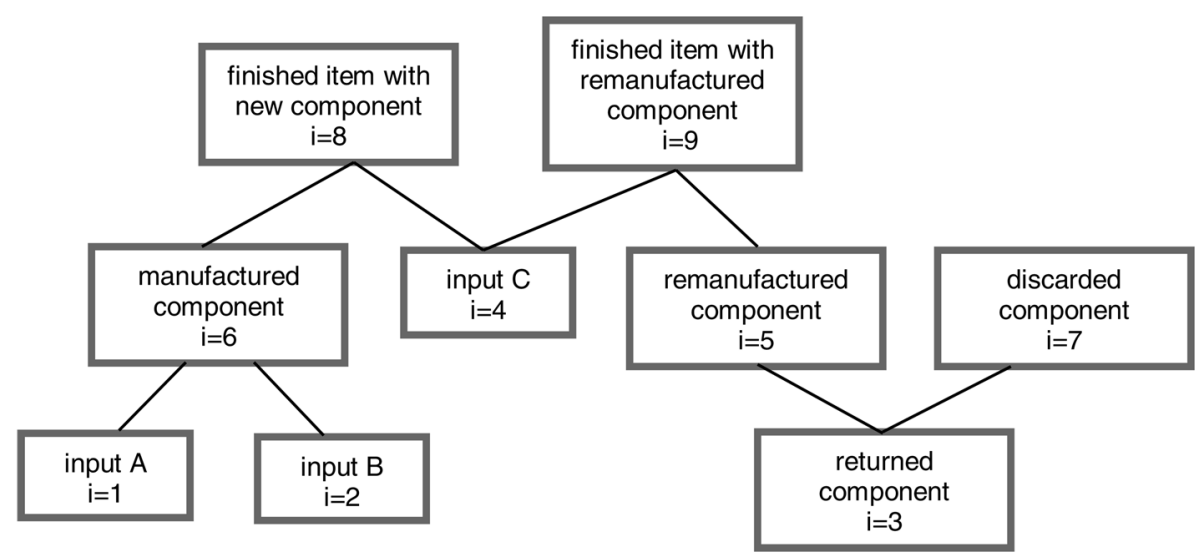

Figure 3 - Modeling structure for the production system

Indices of sets are identified as:

$t \in\{1, \ldots, T\}$ representing one of the time periods of the planning horizon,

$i \in\{1, \ldots, I\}$ representing one of the items involved in the production planning: 
$i=1, \ldots, 6$ are required as input for some other item,

$i=7$ is the disassembled component that is discarded,

$i=8$ is the finished item produced with new component,

$i=9$ is the finished item produced with remanufactured component,

$j \in D(i)$ representing one of the items that are direct successors of item $i$ in BOM.

Using these indices, we define the following deterministic parameters:

$\alpha^{i}$ amount of time needed to produce one unit of item $i$

$\beta^{i}$ amount of time needed to prepare the production of a batch of item $i$

$\gamma_{i}$ procurement/manufacturing/remanufacturing/discarding lead time for item $i$

$\rho \quad$ average rate of assembling component units considered as inserviceable $(0<\rho<1)$

$d_{t}$ external demand for finished item in period $t$, for simplicity, $d_{t}>0 \forall t$,

$h_{t}^{i} \quad$ unitary cost of holding in stock item $i$ in period $t$

$p_{t}^{i} \quad$ unitary cost of purchasing/producing/remanufacturing/discarding item $i$ in period $t$

$q_{t}^{i} \quad$ set-up cost of purchasing/producing/remanufacturing/discarding item $i$ in period $t$

$r^{i j} \quad$ amount of item $i$ required to make one unit of item $j$

$C_{t}$ amount of returned product that is available in period $t$

$K_{t}$ maximum available unit capacity for remanufacturing disassembled component in period $t$

$L_{t} \quad$ maximum available time capacity for manufacturing new component and finished item in period $t$

$M_{t}^{i}$ upper bound on the units of item $i$ that are purchased/produced/discarded in period $t$

$U_{t}^{i}$ upper bound on storage capacity of units of item $i$ in period $t$

$V_{t}$ upper bound on storage capacity of units of serviceable component in period $t$.

The decision variables are:

$x_{t}^{i} \quad$ amount of item $i$ purchased/produced/remanufactured/discarded in period $t$

$y_{t}^{i} \quad$ indicate if item $i$ is purchased/produced/remanufactured/discarded or not in period $t$

$s_{t}^{i} \quad$ amount of item $i$ stocked at the end of period $t$

$p d_{t}^{i}$ (auxiliary variable) part of the demand for finished item that is satisfied with new $(i=8)$ or recovered $(i=9)$ component in period $t$. 
The proposed MRP model is formulated as follows:

$$
\begin{aligned}
& \operatorname{minimize} \sum_{i \in I} \sum_{t \in T} p_{t}^{i} x_{t}^{i}+q_{t}^{i} y_{t}^{i}+h_{t}^{i} s_{t}^{i} \\
& \text { subject to } s_{t-1}^{i}+x_{t-\gamma^{i}}^{i}=\sum_{j \in D(i)} r^{i j} x_{t}^{j}+s_{t}^{i} \quad i=1, \ldots, 6, \forall t \\
& s_{t-1}^{i}+x_{t-\gamma^{i}}^{i}=s_{t}^{i} \quad i=7, \forall t \\
& s_{t-1}^{i}+x_{t-\gamma^{i}}^{i}=p d_{t}^{i}+s_{t}^{i} \quad i=8,9, \forall t \\
& \sum_{i=8}^{9} p d_{t}^{i}=d_{t} \\
& \forall t \\
& x_{t}^{i} \leq M_{t}^{i} y_{t}^{i} \\
& \forall i, i \neq 3,5, \forall t \\
& x_{t}^{i}=C_{t} \\
& i=3, \forall t \\
& x_{t}^{i} \leq K_{t} y_{t}^{i} \\
& i=5, \forall t \\
& \sum_{i}\left(\alpha^{i} x_{t}^{i}+\beta^{i} y_{t}^{i}\right) \leq L_{t} \\
& i=6,8,9, \forall t \\
& \sum_{t \in T} x_{t}^{i}=\rho\left(\sum_{t \in T} C_{t}\right) \\
& i=7 \\
& s_{0}^{i}=0 \\
& \forall i, i \neq 3 \\
& s_{0}^{i}=U_{0}^{i} \\
& i=3 \\
& s_{t}^{i} \leq U_{t}^{i} \\
& \forall i, i \neq 5,6, \forall t \\
& \sum_{i=5}^{6} s_{t}^{i} \leq V_{t} \\
& x \in \mathbb{R}_{+}^{I T}, s \in \mathbb{R}_{+}^{I(T+1)}, y \in\{0,1\}^{I T}, \\
& p d \in \mathbb{R}_{+}^{2 T}
\end{aligned}
$$

where the goal is to minimize the total production and inventory costs (1), and to satisfy the internal demands (2)-(3) and the non-segmented external demand (4)-(5). Although there is no demand for the discarded component, constraints (3) guarantee the flow conservation of this item. In (2) and subsequent occurrences, the notation $x_{t-\gamma^{i}}^{i}$ represents the amount of item $i$ that is delivered in period $t$ by its associated industrial activity, which started in period $t-\gamma^{i}$. As the arrival of the returned product is not considered as an activity in this study, the corresponding lead time is set to zero. Constraints (6) represent the set-up enforcement. The value of $M_{t}^{i}$ should be sufficient large to ensure the satisfaction of all internal or external demand for item $i$ in period $t$; a practical option for the finished item is to set $M_{t}^{i}=\sum_{k=t}^{T} d_{k}^{i}$. Constraints (7) represent the 
availability of units of the returned product during the planning horizon. The constraint capacity on the remanufacturing and manufacturing resources is given by (8) and (9), respectively. Constraint (10) gives the total amount of returned component that is discarded during the planning horizon, which is determined by the average rate $\rho$. Constraints (11) assure that there are no initial inventories, except for the returned product (12), whereas constraints (13) give an upper bound to the storage of units of the items during the planning horizon, including the serviceable component (14). Decision variables are defined in (15).

Even though the amount of units of the returned product $\left(C_{t}\right)$ is known in advance at the beginning and for each period of the planning horizon, it is appropriate to define a decision variable to quantify the amount of units of the returned component that is used by an optimal production plan $\left(x_{t}^{3}\right)$, and consequently know the amount that is recovered $\left(x_{t}^{5}\right)$, the amount that is discarded $\left(x_{t}^{7}\right)$ and stocked $\left(s_{t}^{3}\right)$. This would help future strategic decisions in terms of reverse logistics, such as, for example, augmenting the capacity of the recovering process and evaluating the costs of the recovering process. Also, condition (10) refers to disposal decisions along the planning horizon, which avoids the execution of the disposal activity every single period, whenever possible.

\subsection{Problem and computational complexity}

The proposed MRP model has $I+(I+17) T+1$ functional constraints and $I+(3 I+2) T$ variables, resulting in a problem complexity of $O(I T) \times O(I T)$. Optimization problems that are modeled as the proposed MRP are in the class of mixed integer programming (MIP).

Consider the basic MRP model as given, for example, in Pochet \& Wolsey (2006). Using the notation already defined, it is formulated as follows

$$
\begin{array}{lll}
\operatorname{minimize} & \sum_{i \in I} \sum_{t \in T} p_{t}^{i} x_{t}^{i}+q_{t}^{i} y_{t}^{i}+h_{t}^{i} s_{t}^{i} & \\
\text { subject to } & s_{t-1}^{i}+x_{t-\gamma^{i}}^{i}=d_{t}^{i}+\sum_{j \in D(i)} r^{i j} x_{t}^{j}+s_{t}^{i} & \\
& x_{t}^{i} \leq M_{t}^{i} y_{t}^{i} & \forall i, \forall t \\
& \sum_{i} \alpha^{i k} x_{t}^{i}+\beta^{i k} y_{t}^{i} \leq L_{t}^{k} & \\
& x \in \mathbb{R}_{+}^{I T}, s \in \mathbb{R}_{+}^{I(T+1)}, y \in\{0,1\}^{I T} . &
\end{array}
$$

Based on the works Britan \& Yanasse (1982), Karmarkar \& Nambimadom (1996) and Pochet \& Wolsey (2006), it is known that the basic MRP model is a particular case of the LS-C problem, that is, the lot sizing problem with one item in BOM and capacity on the production resources over a time horizon. Regarding LS-C, it can be shown that it is a particular case of the 0-1 knapsack problem [PW06], which in turn is a NP-hard problem, [KN96] apud Garey \& Johnson (1979). So, by reducibility, we have that the MRP basic model (16)-(20) is a NP-hard problem. As the MRP basic model (16)-(20) is a particular case of the MRP model (1)-(15), in the sense that new constraints were added to model (1)-(15) in relation to model (16)-(20), the problem 
addressed in this work is NP-hard. (Note that we can always set to zero the parameters of the additional constraints of the model (1)-(15) to get model (16)-(20).)

\section{COMPUTATIONAL EXPERIMENTS}

In this section, we report computational experiments with the proposed MRP model. To this end, an arranged instance of the studied production planning problem was tested using the solver CPLEX 12.3 with the standard choice for solving mixed integer programming problems, in the software package AIMMS version 3.12. In this CPLEX version, the solution method for mixed integer linear programming problems is the branch-and-cut algorithm.

The first computational test considers the following arranged parameter selection:

$$
\begin{gathered}
\alpha=(0,0,0,0,0,30,0,20,20) ; \quad \beta=(0,0,0,0,0,90,0,60,60) ; \\
\gamma=(1,1,0,1,1,1,1,1,1) ; \quad \rho=0.25 ; \quad d=(10,13,16,14,15) ; \\
h=(1,2,2,1,3,3,3,5,5) \forall t ; \quad p=(2,1,0,5,10,16,5,20,20) \forall t ; \\
q=(20,10,0,50,200,200,150,220,220) \forall t ; \\
r^{16}=2, \quad r^{26}=1, \quad r^{35}=1, \quad r^{37}=1, \quad r^{48}=2, \quad r^{49}=2, \quad r^{59}=1, \quad r^{68}=1 ; \\
C=(10,8,10,8,8) ; \quad K_{t}=20 \forall t ; \quad L_{t}=2200 \forall t ; \quad M_{t}^{i}=100 \forall i, \forall t ; \quad U_{t}^{i}=30 \forall i, \forall t,
\end{gathered}
$$

and $U_{0}^{3}=30 ; \quad V_{t}=30 \forall t$.

Observe that the demand for the final product $(d)$ is given for 5 periods ahead. But, to procure the needed inputs and process some production activities in advance, so that the demand for the final product in period $t=1$ is satisfied, at least two periods should be anticipated, totalizing the planing horizon of at least 7 periods ahead $(T=7)$. Recall that the lead time is at most 1 period long.

The corresponding optimal production plan is described in Table 1, and the main information from the final report generated by CPLEX is showed in Table 2. It can be observed that many of the production activities have to be anticipated so that the demand for the final product could be satisfied in each corresponding period. For example, take the demand for the final product at $t=1, d_{1}=10$, as the lead time for assembling the final product with recovered component is $\gamma^{9}=1$, this activity had to be anticipated, as we observe that $x_{0}^{9}=10$, to get the optimal total cost.

\subsection{Possible scenarios}

The first scenario to be analyzed, corresponding to the second computational test, considers limitation on the availability of the inputs A, B and C. This is a common situation faced by manufactures, when the suppliers could not deliver the total amount of raw materials of an order placed. In this scenario, the arranged parameter selection is identical to the first computational test as well as the model, except for the conditions that are additionally introduced into the model: $x_{t}^{1} \leq 18, x_{t}^{2} \leq 10$ and $x_{t}^{4} \leq 28 \forall t$, which means that, per period $t$, the total amount available for 
Table 1 - Optimal values for $x_{t}^{i}$ and $s_{t}^{i}$ of Test 1 - Given instance.

\begin{tabular}{|c|ccccccc||}
\hline$x_{t}^{i}$ & \multicolumn{7}{|c||}{$t$} \\
\hline$i$ & -1 & 0 & 1 & 2 & 3 & 4 & 5 \\
\hline 1 & 56 & 0 & 0 & 0 & 0 & 0 & 0 \\
2 & 28 & 0 & 0 & 0 & 0 & 0 & 0 \\
3 & 0 & 0 & 10 & 8 & 10 & 8 & 8 \\
4 & 22 & 56 & 0 & 58 & 0 & 0 & 0 \\
5 & 20 & 0 & 0 & 20 & 0 & 0 & 0 \\
6 & 0 & 28 & 0 & 0 & 0 & 0 & 0 \\
7 & 0 & 0 & 0 & 0 & 0 & 0 & 11 \\
8 & 0 & 0 & 28 & 0 & 0 & 0 & 0 \\
9 & 0 & 11 & 0 & 0 & 29 & 0 & 0 \\
\hline
\end{tabular}

\begin{tabular}{||c|ccccccc|}
\hline$s_{t}^{i}$ & & & & $t$ & & & \\
\hline$i$ & -1 & 0 & 1 & 2 & 3 & 4 & 5 \\
\hline 1 & 0 & 0 & 0 & 0 & 0 & 0 & 0 \\
2 & 0 & 0 & 0 & 0 & 0 & 0 & 0 \\
3 & 10 & 10 & 20 & 8 & 18 & 26 & 23 \\
4 & 0 & 0 & 0 & 0 & 0 & 0 & 0 \\
5 & 0 & 9 & 9 & 9 & 0 & 0 & 0 \\
6 & 0 & 0 & 0 & 0 & 0 & 0 & 0 \\
7 & 0 & 0 & 0 & 0 & 0 & 0 & 0 \\
8 & 0 & 0 & 0 & 16 & 0 & 0 & 0 \\
9 & 0 & 0 & 1 & 0 & 0 & 15 & 0 \\
\hline
\end{tabular}

Table 2 - Final Report for Test 1 - Given instance.

\begin{tabular}{ll}
\hline optimal function value & 5144 \\
number of functional constraints & 198 \\
number of real variables & 140 \\
number of integer variables & 56 \\
running time (s) & 0.09 \\
number of iterations & 501 \\
number of generated nodes & 41 \\
\hline
\end{tabular}

procurement of input $\mathrm{A}$ is 18 , for input $\mathrm{B}$ is 10 , and for input $\mathrm{C}$ is 28 . The corresponding optimal production plan is described in Table 3 , and the main information from the final report generated by CPLEX is showed in Table 4.

Table 3 - Optimal values for $x_{t}^{i}$ and $s_{t}^{i}$ of Test 2 - Scenario 1.

\begin{tabular}{|c|ccccccc||}
\hline$x_{t}^{i}$ & \multicolumn{7}{|c||}{$t$} \\
\hline$i$ & -1 & 0 & 1 & 2 & 3 & 4 & 5 \\
\hline 1 & 4 & 18 & 0 & 0 & 0 & 0 & 0 \\
2 & 1 & 10 & 0 & 0 & 0 & 0 & 0 \\
3 & 0 & 0 & 10 & 8 & 10 & 8 & 8 \\
4 & 28 & 28 & 24 & 28 & 28 & 0 & 0 \\
5 & 10 & 18 & 0 & 14 & 15 & 0 & 0 \\
6 & 0 & 0 & 11 & 0 & 0 & 0 & 0 \\
7 & 0 & 0 & 0 & 0 & 0 & 0 & 11 \\
8 & 0 & 0 & 0 & 11 & 0 & 0 & 0 \\
9 & 0 & 10 & 18 & 0 & 14 & 15 & 0 \\
\hline
\end{tabular}

\begin{tabular}{||c|ccccccc|}
\hline$s_{t}^{i}$ & \multicolumn{7}{|c|}{$t$} \\
\hline$i$ & -1 & 0 & 1 & 2 & 3 & 4 & 5 \\
\hline 1 & 0 & 4 & 0 & 0 & 0 & 0 & 0 \\
2 & 0 & 1 & 0 & 0 & 0 & 0 & 0 \\
3 & 20 & 2 & 12 & 6 & 1 & 9 & 6 \\
4 & 0 & 8 & 0 & 2 & 2 & 0 & 0 \\
5 & 0 & 0 & 0 & 0 & 0 & 0 & 0 \\
6 & 0 & 0 & 0 & 0 & 0 & 0 & 0 \\
7 & 0 & 0 & 0 & 0 & 0 & 0 & 0 \\
8 & 0 & 0 & 0 & 0 & 0 & 0 & 0 \\
9 & 0 & 0 & 0 & 5 & 0 & 0 & 0 \\
\hline
\end{tabular}


Table 4 - Final Report for Test 2 - Scenario 1.

\begin{tabular}{ll}
\hline optimal function value & 5611 \\
number of functional constraints & 219 \\
number of real variables & 140 \\
number of integer variables & 56 \\
running time (s) & 0.06 \\
number of iterations & 606 \\
number of generated nodes & 60 \\
\hline
\end{tabular}

The second analyzed scenario considers a maximal amount of 10 units of the finished item that can be assembled with the recovered component. This situation can occur, when resources of the recovering line is limited, due to temporary and casual events. In this case, we introduce the following constraint into the model and consider the instance parameters of the first computational test: $x_{t}^{9} \leq 10 \forall t$. The corresponding optimal production plan is described in Table 5, and the main information from the final report generated by CPLEX is showed in Table 6.

Table 5-Optimal values for $x_{t}^{i}$ and $s_{t}^{i}$ of Test 3 - Scenario 2.

\begin{tabular}{|c|ccccccc||}
\hline$x_{t}^{i}$ & & & & $t$ & & & \\
\hline$i$ & -1 & 0 & 1 & 2 & 3 & 4 & 5 \\
\hline 1 & 56 & 0 & 0 & 0 & 0 & 0 & 0 \\
2 & 28 & 0 & 0 & 0 & 0 & 0 & 0 \\
3 & 0 & 0 & 10 & 8 & 10 & 8 & 8 \\
4 & 20 & 76 & 0 & 40 & 0 & 0 & 0 \\
5 & 20 & 0 & 0 & 20 & 0 & 0 & 0 \\
6 & 0 & 28 & 0 & 0 & 0 & 0 & 0 \\
7 & 0 & 0 & 0 & 0 & 0 & 0 & 11 \\
8 & 0 & 0 & 28 & 0 & 0 & 0 & 0 \\
9 & 0 & 10 & 10 & 0 & 10 & 10 & 0 \\
\hline
\end{tabular}

\begin{tabular}{||c|ccccccc|}
\hline$s_{t}^{i}$ & \multicolumn{1}{|c|}{$t$} \\
\hline$i$ & -1 & 0 & 1 & 2 & 3 & 4 & 5 \\
\hline 1 & 0 & 0 & 0 & 0 & 0 & 0 & 0 \\
2 & 0 & 0 & 0 & 0 & 0 & 0 & 0 \\
3 & 10 & 10 & 20 & 8 & 18 & 26 & 23 \\
4 & 0 & 0 & 0 & 0 & 20 & 0 & 0 \\
5 & 0 & 10 & 0 & 0 & 10 & 0 & 0 \\
6 & 0 & 0 & 0 & 0 & 0 & 0 & 0 \\
7 & 0 & 0 & 0 & 0 & 0 & 0 & 0 \\
8 & 0 & 0 & 0 & 25 & 9 & 5 & 0 \\
9 & 0 & 0 & 0 & 0 & 0 & 0 & 0 \\
\hline
\end{tabular}

Table 6 - Final Report for Test 3 - Scenario 2.

\begin{tabular}{ll}
\hline optimal function value & 5618 \\
number of functional constraints & 205 \\
number of real variables & 140 \\
number of integer variables & 56 \\
running time (s) & 0.02 \\
number of iterations & 124 \\
number of generated nodes & 7 \\
\hline
\end{tabular}


The third analyzed scenario considers that, among the units of finished item that are demanded per each period of the planning horizon, 5 units is the minimal amount that should be assembled with new manufactured component. This scenario could be generated by a client that is not confident on the recovering process. In this case, we introduce the following constraint into the model and consider also the instance parameters of the first computational test: $x_{t}^{8} \geq 5 \forall t$. The corresponding optimal production plan is described in Table 7, and the main information from the final report generated by CPLEX is showed in Table 8 .

Table 7 - Optimal values for $x_{t}^{i}$ and $s_{t}^{i}$ of Test 4 - Scenario 3.

\begin{tabular}{|c|ccccccc||}
\hline$x_{t}^{i}$ & & & & $t$ & & & \\
\hline$i$ & -1 & 0 & 1 & 2 & 3 & 4 & 5 \\
\hline 1 & 66 & 0 & 0 & 0 & 0 & 0 & 0 \\
2 & 33 & 0 & 0 & 0 & 0 & 0 & 0 \\
3 & 0 & 0 & 10 & 8 & 10 & 8 & 8 \\
4 & 46 & 0 & 64 & 0 & 36 & 0 & 0 \\
5 & 20 & 0 & 20 & 0 & 0 & 0 & 0 \\
6 & 0 & 33 & 0 & 0 & 0 & 0 & 0 \\
7 & 0 & 0 & 0 & 0 & 0 & 0 & 11 \\
8 & 0 & 0 & 5 & 5 & 5 & 13 & 5 \\
9 & 0 & 18 & 0 & 22 & 0 & 0 & 0 \\
\hline
\end{tabular}

\begin{tabular}{||c|ccccccc|}
\hline$s_{t}^{i}$ & & & & & & & \\
\hline$i$ & -1 & 0 & 1 & 2 & 3 & 4 & 5 \\
\hline 1 & 0 & 0 & 0 & 0 & 0 & 0 & 0 \\
2 & 0 & 0 & 0 & 0 & 0 & 0 & 0 \\
3 & 10 & 10 & 0 & 8 & 18 & 26 & 23 \\
4 & 0 & 10 & 0 & 10 & 0 & 10 & 0 \\
5 & 0 & 2 & 2 & 0 & 0 & 0 & 0 \\
6 & 0 & 0 & 28 & 23 & 18 & 5 & 0 \\
7 & 0 & 0 & 0 & 0 & 0 & 0 & 0 \\
8 & 0 & 0 & 0 & 0 & 5 & 2 & 0 \\
9 & 0 & 0 & 8 & 0 & 6 & 0 & 0 \\
\hline
\end{tabular}

Table 8 - Final Report for Test 4 - Scenario 3.

\begin{tabular}{ll}
\hline optimal function value & 6367 \\
number of functional constraints & 203 \\
number of real variables & 140 \\
number of integer variables & 56 \\
running time (s) & 0.05 \\
number of iterations & 211 \\
number of generated nodes & 9 \\
\hline
\end{tabular}

The fourth analyzed scenario considers the case in which the remanufacturing line will be out of order during the periods $t=2,3$ and 4 for preventive maintenance. In this case, we introduce the following constraint into the model and also consider the instance parameters of the first computational test: $x_{t}^{9}=0 \forall t=2,3,4$. The corresponding optimal production plan is described in Table 9, and the main information from the final report generated by CPLEX is showed in Table 10. 
Table 9-Optimal values for $x_{t}^{i}$ and $s_{t}^{i}$ of Test 5 - Scenario 4.

\begin{tabular}{|c|ccccccc||}
\hline$x_{t}^{i}$ & & & & $t$ & & & \\
\hline$i$ & -1 & 0 & 1 & 2 & 3 & 4 & 5 \\
\hline 1 & 0 & 76 & 0 & 0 & 0 & 0 & 0 \\
2 & 0 & 38 & 0 & 0 & 0 & 0 & 0 \\
3 & 0 & 0 & 10 & 8 & 10 & 8 & 8 \\
4 & 60 & 0 & 76 & 0 & 0 & 0 & 0 \\
5 & 15 & 15 & 0 & 0 & 0 & 0 & 3 \\
6 & 0 & 0 & 38 & 0 & 0 & 0 & 0 \\
7 & 0 & 0 & 0 & 0 & 0 & 11 & 0 \\
8 & 0 & 0 & 0 & 38 & 0 & 0 & 0 \\
9 & 0 & 15 & 15 & 0 & 0 & 0 & 0 \\
\hline
\end{tabular}

\begin{tabular}{||c|ccccccc|}
\hline$s_{t}^{i}$ & & & & $t$ & & & \\
\hline$i$ & -1 & 0 & 1 & 2 & 3 & 4 & 5 \\
\hline 1 & 0 & 0 & 0 & 0 & 0 & 0 & 0 \\
2 & 0 & 0 & 0 & 0 & 0 & 0 & 0 \\
3 & 15 & 0 & 10 & 18 & 28 & 25 & 30 \\
4 & 0 & 30 & 0 & 0 & 0 & 0 & 0 \\
5 & 0 & 0 & 0 & 0 & 0 & 0 & 0 \\
6 & 0 & 0 & 0 & 0 & 0 & 0 & 0 \\
7 & 0 & 0 & 0 & 0 & 0 & 0 & 11 \\
8 & 0 & 0 & 0 & 0 & 29 & 15 & 0 \\
9 & 0 & 0 & 5 & 7 & 0 & 0 & 0 \\
\hline
\end{tabular}

Table 10 - Final Report for Test 5 - Scenario 4.

\begin{tabular}{ll}
\hline optimal function value & 5558 \\
number of functional constraints & 201 \\
number of real variables & 140 \\
number of integer variables & 56 \\
running time (s) & 0.06 \\
number of iterations & 368 \\
number of generated nodes & 42 \\
\hline
\end{tabular}

\subsection{Computational analysis}

Let us now go back to the given instance to show some analysis of the computational tests with respect to the modification of some parameters values. First, consider the variation on the data for $\rho$, the average rate of disassembled component units considered as inserviceable, showed in Table 11.

Table 11 - The optimal total costs in relation to the modification of $\rho$.

\begin{tabular}{cc}
\hline$\rho$ & $\begin{array}{c}\text { optimal function } \\
\text { value }\end{array}$ \\
\hline 0.10 & 5124.2 \\
$0.25 *$ & 5144 \\
0.50 & 5177 \\
0.75 & 5210 \\
\hline *indicates the original value
\end{tabular}


From Table 11, we verify that the optimal total costs decrease when we reduce the value of $\rho$ $(\rho=0.10)$ from its original value $(\rho=0.25)$. Since the disposal activities are reduced the cost of these activities is lowered, which is reflected in the optimal total costs. When we increase the value of $\rho(\rho=0.50,075)$ from its original value $(\rho=0.25)$, we verify that the corresponding optimal costs also increase, due to the fact that more disposal activities are processed and the inventory level of inserviceable component is larger along the planning period.

Consider now the modification on the values of the unitary manufacturing cost $\left(p_{6}\right)$ and the unitary recovering cost $\left(p_{5}\right)$ of the main component. Recall that in the given instance the values of the unitary manufacturing cost of the final product with new or recovered component are equal $\left(p_{8}=p_{9}\right)$. The obtained optimal function values are reported in Table 12 .

Table 12 - The optimal total costs in relation to modification of $p_{5}$ and $p_{6}$.

\begin{tabular}{ccc}
\hline$p_{5}$ & $p_{6}$ & $\begin{array}{c}\text { optimal function } \\
\text { value }\end{array}$ \\
\hline 10 & 22 & 5262 \\
$10 *$ & $16^{*}$ & 5144 \\
10 & 10 & 4976 \\
16 & 10 & 5216 \\
22 & 10 & 5456 \\
\hline \multicolumn{3}{c}{ *indicates the original value }
\end{tabular}

In relation to the given instance data, $p_{5}=10$ and $p_{6}=16$, we observe that it is preferable to assemble the finished product with recovered component than manufactured component. From Table 12, we verify that, for setting $p_{5}=10$ and increasing $p_{6}=10,16,22$, we get larger optimal function values. When $p_{6}=10$ is fixed and $p_{5}=10,16,22$ increases, we get also larger optimal function values. For all cases showed in Table 12, the total units of recovered component is 40 and of the manufactured component is 28 along the planning period, matching the amounts obtained for the given instance, except for the case $p_{5}=10$ and $p_{6}=22$, where the total units of recovered component is 55 and of the manufactured component is 13.

Consider now the variation of the setup costs data for the items 5 and 6 (recovered and manufactured component, resp.) and the items 8 and 9 (finished product with manufactured and recovered component, resp.) that could influence the optimal function value, as presented in Table 13.

From Table 13, we verify that reducing the setup costs causes a decreasing of the optimal function values. In particular, we verify that, halving $q_{5}$ and $q_{6}$, the decisions on the quantities to produce, along the planning period, of the finished product with recovered component change from 40 to 48 , whereas the quantities of the finished product with manufactured component change from 28 to 20 , meaning that it is still preferable to assemble finished product with recovered component. We could see that, when the values of $q_{8}$ and $q_{9}$ are half of their original values, the total units of the finished product assembled with recovered component changes from 40 to 55, and the total 
Table 13 - The optimal total costs in relation to modification of $q_{5}, q_{6}, q_{8}$, and $q_{9}$.

\begin{tabular}{ccccc}
\hline$q_{5}$ & $q_{6}$ & $q_{8}$ & $q_{9}$ & $\begin{array}{c}\text { optimal function } \\
\text { value }\end{array}$ \\
\hline $200 *$ & $200 *$ & $220 *$ & $220 *$ & 5144 \\
100 & 100 & 220 & 220 & 4766 \\
200 & 200 & 110 & 110 & 4744 \\
100 & 100 & 110 & 110 & 4344 \\
\hline \multicolumn{4}{c}{ *indicates the original value }
\end{tabular}

units of the finished product assembled with manufactured component changes from 28 to 13 , which shows also a preference to assemble finished product with recovered component. In case $q_{5}, q_{6}, q_{8}$, and $q_{9}$ are reduced in half in respect to their original values, we get the same result for the amount of the finished product as when $q_{8}$ and $q_{9}$ are reduced in half.

We also analyzed the sensitivity of the optimal function value for the given instance in relation to the variation of $L_{t}$, (originally set to 2200). We verified that for $1400 \leq L_{t} \leq 2200$, the optimal function value does not chance, with some differences on the inventory decisions along the planning period. The computational efforts do change; for $L_{t}=1600$, the solver finds the solution with minimal generated nodes (12) and minimal number of iterations (297), whereas for $L_{t}=2200$, the solver finds the solution with 41 generated nodes and 501 iterations, as we can see in Table 2.

The same analysis was performed with respect to the value of $U_{t}$, for $t \neq 0$, originally set to $U_{t}=30$. For $26 \leq U_{t} \leq 30$, the optimal function value does not change, but the inventory decisions do change along the planning period.

\section{CONCLUSION AND FINAL COMMENTS}

This work deals with the use of a technique of Operations Research to support decisions of industrial organizations that practice reverse logistics in their production systems. Specifically, a mixed integer linear programming model is proposed for the planning of materials requirements (MRP) of a particular production system with recovering and discarding process.

Computational experiments were conducted with the proposed MRP model, considering an arranged instance of the problem and distinct scenarios for the production system, with the main purpose to show its versatility. One can observe that the lowest-cost optimal plan found for the given data shows a preference to assemble the final product with recovered component, since remanufacturing is cheaper than manufacturing a new component for this data (although in some practical production systems the recovering process could be more expensive). This decision is confirmed with the results of the third scenario, that established a minimum amount of units for the finished product that should be assembled with new component, which resulted in a highercost optimal plan. 
The optimal production plan generated with CPLEX solver would help the decision makers to decide how much and when to procure/produce/remanufacture/discard of each item involved in the production system during a time horizon. Complex production systems with remanufacturing can be easily derived from the particular production system presented here.

\section{ACKNOWLEDGMENTS}

The authors are thankful for the valuable suggestions given by the anonymous referees that improved the paper. The first author was partially supported by FAPERJ/CNPq through PRONEX 662199/2010-12 and CNPq Grant 311165/2013-3, whereas the last author was supported by Edital Universal 14/2012, n. 475245/2012-1.

\section{REFERENCES}

[1] Barba-Gutiérrez Y, Adenso-Díaz B \& Gupta SM. 2008. Lot sizing in reverse MRP for scheduling disassembly. International Journal of Production Economics, 111(2): 741-751.

[2] BAYINDIR ZP, ERKIPB N \& GÜLLÜB R. 2007. Assessing the benefits of remanufacturing option under one-way substitution and capacity constraint. Computers \& Operations Research, 34: 487 514.

[3] BitRAn GR \& YANASSE HH. 1982. Computational complexity of the capacitated lot size problem. Management Science, 28(10): 1174-1186.

[4] Brito MP, Dek Ker R \& Flapper SDP. 2005. Reverse Logistics: A Review of Case Studies. In Distribution Logistics: Advanced Solutions To Practical Problems, Lecture Notes in Economics and Mathematical Systems, Bernhard Fleischmann, Andreas Klose Editors. 544: 243-272, Springer.

[5] DePuy GW, Usher JS, WAlker RL \& TAYlor GD. 2007. Production planning for remanufactured products. Production Planning \& Control: The Management of Operations, 18(7): 573-583. DOI: $10.1080 / 0953728070154221$.

[6] Fleischmann M. 2000. Quantitative Models for Reverse Logistics. PhD thesis, Erasmus University Rotterdam, the Netherlands.

[7] Garey MR \& Johnson DS. 1979. Computers and Intractability: A Guide to NP-Completeness, Freeman.

[8] Gotzel C \& Inderfurth K. 2002. Performance of MRP in Product Recovery Systems with Demand, Return and Leadtime Uncertainties. In Quantitative Approaches to Distribution Logistics and Supply Chain Management, Lecture Notes in Economics and Mathematical Systems, 519: 99-114, Springer.

[9] Helmrich MJR, Jans R, Heuvel Wvd \& Wagelmans APM. 2014. Economic lot-sizing with remanufacturing: complexity and efficient formulations. IIE Transactions, Special Issue: Scheduling \& Logistics, $\mathbf{4 6}(1)$.

[10] Inderfurth K \& Jensen T. 1999. Analysis of MRP Policies with Recovery Options. In Modelling and Decisions in Economics: Essays in Honor of Franz Ferschl, Editors: U. Leopold-Wildburger, G. Feichtinger, K.-P. Kistner, 1st edition, Physica-Verlag HD, Heidelberg-New York, 189-228. 
[11] Karmarkar US \& NAmbimadom RS. 1996. Material Allocation in MRP with Tardiness Penalties. Journal of Global Optimization, 9: 453-482.

[12] Lage Junior M \& Godinho Filho M. 2012. Production planning and control for remanufacturing: literature review and analysis. Production Planning \& Control, 23(6): 419-435.

[13] MitRA S. 2012. Inventory management in a two-echelon closed-loop supply chain with correlated demands and returns. Computers \& Industrial Engineering, 62: 870-879.

[14] Paper Industry Association Council of USA. 2013. Paper \& Paperboard Recovery. Available at www.paperrecycles.org/stat_pages/recovery_rate.html.

[15] PIÑEYRO P \& VIERA O. 2010. The economic lot-sizing problem with remanufacturing and one-way substitution. International Journal of Production Economics, 124(2): 482-488.

[16] Piñeyro P \& Viera O. 2012. Analysis of the quantities of the remanufacturing plan of perfect cost. Journal of Remanufacturing, 2(3). Available at: http://www.journalofremanufacturing.com/ content $/ 2 / 1 / 3$.

[17] Pochet Y \& Wolsey LA. 2006. Production Planning by Mixed Integer Programming. Springer Series in Operations Research and Financial Engineering, Springer.

[18] REIMANN M \& ZHANG W. 2013. Joint optimization of new production, warranty servicing strategy and secondary market supply under consumer returns. Pesquisa Operacional, 33(3), Rio de Janeiro, Setp/Dec 2013.

[19] Rogers DS \& TiBBen-LembKe RS. 1999. Going backwards: reverse logistics trends and practices. University of Nevada, Reno, Center for Logistics Management, Reverse Logistics Executive Council.

[20] Schulz T \& FERRETTI I. 2011. On the alignment of lot sizing decisions in a remanufacturing system in the presence of random yield. Journal of Remanufacturing, 1(3). DOI:10.1186/2210-4690-1-3.

[21] SRIVAstaVA SK. 2008. Network design for reverse logistics. Omega, 36: 535-548.

[22] TEUNTER R. 2004. Lot-sizing for inventory systems with product recovery. Computers \& Industrial Engineering, 46: 431-441.

[23] Thierry M, Salomon M, Van Nunen J \& VAn Wassenhove LN. 1995. Strategic issues in product recovery management. California Management Review, 37(2): 114-128. 